\title{
Digital Age Estimation Using Orthopatomograms in Indian Population: A Comparative Study
}

\section{Ramakant Gupta ${ }^{1 *}$, Swati Gupta ${ }^{2}$ and Manju Gupta ${ }^{3}$}

${ }^{1}$ Head and Consultant, Department of Dental Services, Dr. Jatinder Gupta's Gupta

Clinic and Opticals, Haryana, India

${ }^{2}$ Senior Consultant, Oral and Maxillofacial Pathology, Dr. Jatinder Gupta's Gupta Clinic

and Opticals, Haryana, India

${ }^{3}$ Director and Clinic coordinator, Dr. Jatinder Gupta's Gupta Clinic and Opticals,

Haryana, India

*Corresponding Author: Ramakant Gupta, Head and Consultant, Department of

Dental Services, Dr. Jatinder Gupta's Gupta Clinic and Opticals, Haryana, India.
Received: April 02, 2020

Published: June 18, 2020

(C) All rights are reserved by Ramakant

Gupta., et al.

\begin{abstract}
Introduction: Orthopantomographs are non- destructive tool for age estimation by indirectly measuring the rate of secondary dentine deposition. Cameriere.,et al. introduced one such Europeanpopulation based method and modified by researchers for Indian population; but their applicability is yet to be assessed.

Aim and Objective: To assess the applicability of Cameriere's method and its modification by Jeevan.,et al. Bebshet.,et al. for mandibular canines and Sexena, Jeevan.,et al,Juneja.,et al. for maxillary canines in an Indian population in Faridabad district of Haryana by evaluating the pulp/tooth ratio in canines on right side using digital orthopantomographs.

Materials and Methods: Digital orthopantomographs of 60 patients (30 males, 30 females, 16- 72 years) were selected by simple random sampling. Pulp and tooth areas were measured and their ratio was computed using computer software. Age was calculated using the Cameriere's method and its modification by Jeevan., et al, Bebshet.,et al, Sexena, Juneja., et al. Mean absolute error (MAE) for each method was calculated for each method.

Results: The present study showed no recognisable difference in dental age assessed by all the methods. Overestimation of age with $\mathrm{MAE}< \pm 10$ years was found in Indian population using all the methods.

Conclusion: Jeevan's formula revealed least MAE and slightly more percentage of cases with errors $< \pm 10$ years i.e. acceptable" age estimates than others, therefore, itis more applicable for Indian population.

Keywords: Dental Age Estimation; Digital Orthopantomographs; Radiographs
\end{abstract}

\section{Abbreviations}

AR: Pulp to Tooth Area Ratio;CEJ: Cemento-Enamel Junction; RIC: Digital Radiographic Image of Canine; L: Magnetic Lasso Tool; MAE: Mean Absolute Error

\section{Introduction}

Age estimation is one of the preliminary and often crucial steps in forensics. It is imperative not only for identification of deceased but is also in livings during various civil cases viz. marriage, attainment of majority, competency as a witness, senior citizen concession, retirement benefits, etc. or criminal cases such as juvenile offenders, kidnaps, rape etc. The different age estimating systems available commonly use indicators like bone maturation, second- ary sex characteristics, height, weight, etc [1]. Ideally, age estimation should be a conclusion of the critical outcome of all the available possible data put into the systems and considered separately, if the forensic situation provides enough such evidence [1]. However, now-a-days dental age estimating systems have gained lot of impetus and focus of attention by researchers worldwide owing to its lower variability, the ease of the procedure, and especially when the availability of other evidence/remains is scarce [1-4].

The different methods of dental age estimation include morphological, histological, biochemical and radiological assessment of teeth [5-7]. However, most methods require extraction of teeth thus, making them inappropriate to use in living individuals [5- 
7]. Therefore, use of radiographs for age estimation is practical, non- destructive, reliable and relatively simple method to use in not only in living individuals but even in deceased and in skeletal remains[1-8]. Radiographic methods of age estimation using teeth usually depend on developmental stages of teeth especially in children [1,5].In adults the amount of deposition of secondary dentin as depicted by reduction in pulp area is used for the purpose [9].

An age estimation method by indirectly measuring secondary dentine deposition on radiographs was initially introduced by Kvaal.,et al [10]. The authors proposed a numerouslength and width measurements of tooth and pulp [10].A similar method was put forth by Cameriere's.,et al.[11] based on radiographic estimation of pulp to tooth area ratio (AR) in canines. Canines are favoured since they are single rooted with largest pulp area providing ease of analysis $[9,11]$. In addition, they are frequently present in older age groups and have less chance for wear as compared to other anterior teeth $[9,11]$. This European population based method by Cameriere.,et al.became one of the widely accepted dental age estimating method due to its ease and minimal variance $[9,11,12]$. Initially, Conventional intra-oral periapical radiographs and orthopantomographs were used for the purpose $[9,11]$. However, with the advent of digital approaches in oral diagnostics such as digital orthopantomographs and radiovisiographs and also the use of digital techniques in forensic investigations i.e. computer aided image analysis have further revolutionized the dental age estimating systems, and have eased them and enhanced their simplicity, accuracy, reliability and reproducibility $[3,7,13,14]$.

With the increased demands for population-specific equations to enhance the age prediction, Jeevan.,et al.[13], Bebshet.,et al.[15] modified the Cameriere's equation for mandibular canines and Sexena[16], Jeevan.,et al.[13], Juneja.,et al.[17] modified the Cameriere's equation for maxillary canines and gave the Indian population specific equation. However, to the best of our knowledge, no studies till now have been conducted to assess the applicability of these methods for age estimation on the Indian population in the Faridabad district of Haryana.

The present study sought to assess the applicability and to compare dental age estimation by Cameriere's method [11] and its modification by Jeevan.,et al.[13], Bebshet.,et al.[15] for mandibular canines and Sexena[16], Jeevan.,et al.[13], Juneja.,et al.[17] for maxillary canines in an Indian population in Faridabad district of Haryana by evaluating the pulp/tooth ratio in canines on right side using digital orthopantomographs.

\section{Materials and Methods}

In this cross-sectional study, digital orthopantomographs of 100 patients (50 males, 50 females) were obtained from the pretreatment records in various private dental clinics all over Faridabad district of Haryana, India. The study sample was selected by simple random sampling. The study was reviewed and approved in accordance with local legislation. All procedures performed were in accordance to ethical standards of the responsible committee on human experimentation (institutional and national) and as per the Declaration of Helsinki (1964, revised in 2013).

The digital orthopantomographs of patients within 16- 72 years of age included were and divided into four groups each with an interval of 14 years (Table 1). All patients included were with wellknown chronological age. The exclusion criteria were: canines with caries, large areas of enamel overlap between neighbouring teeth, radio-opaque fillings, crown, and any other pathologic changes like attrition, abrasion, fracture, impaction, endodontically treated, any congenital anomalies or bone lesions, any systemic illness.

Following Cameriere., et al. [11] and its modification by Bebshet,et al.[15], the radiographic images were photo edited. The radiographic images were saved as high resolution JPEG files and imported to Adobe Photoshop 7 image-editing software programme (Adobe Systems Inc., Mountain View, CA, USA). The tooth's long axis was aligned vertically using the measure tool. Then, eleven horizontal lines were marked on the tooth using Photoshop's inbuilt line tool (Figure 1). The lines were marked at the cusp tip, at two levels corresponding to the maximum curvature of crown on the mesial and distal side, as well as the cemento-enamel junction (CEJ); from the CEJ, lines were marked apically at every one-eighth increments up to the root apex, the exception being the one-eighth increment immediately apical to the CEJ. Marking of the lines, particularly the ones based on root length, allowed for designation of points at specific regions of the tooth and pulp perimeters on all radiographs.The images were again saved as high resolution JPEG files. The digital radiographic image of canine (RIC) was again opened and processed using Adobe Photoshop 7. Using the magnetic lasso tool (L) provided in this program, the outline of the canine and its pulp chamber were traced by marking twenty points on the outline of the tooth, at the junction of the marked horizontal lines, a minimum of 10 points were also marked on the pulp outline although up to 18 points were marked in some cases. These points were connected and area was obtained for both tooth and 


\begin{tabular}{|c|c|c|}
\hline Age (years) & No. of males & No. of females \\
\hline $16-30$ & 15 & 16 \\
\hline $31-44$ & 13 & 12 \\
\hline $45-58$ & 10 & 11 \\
\hline $59-72$ & 12 & 11 \\
\hline Total & 50 & 50 \\
\hline
\end{tabular}

pulp (Figure 2 and 3). Later, the morphological variable, i.e. pulp/ tooth area ratio (AR) was derived.

A random sample of 25 orthopantomographs was used for repeat measurements and the values were subjected to a paired $t$ test to assess potential intra- and inter-observer error.

Table 1: Age and gender distribution of the study groups.

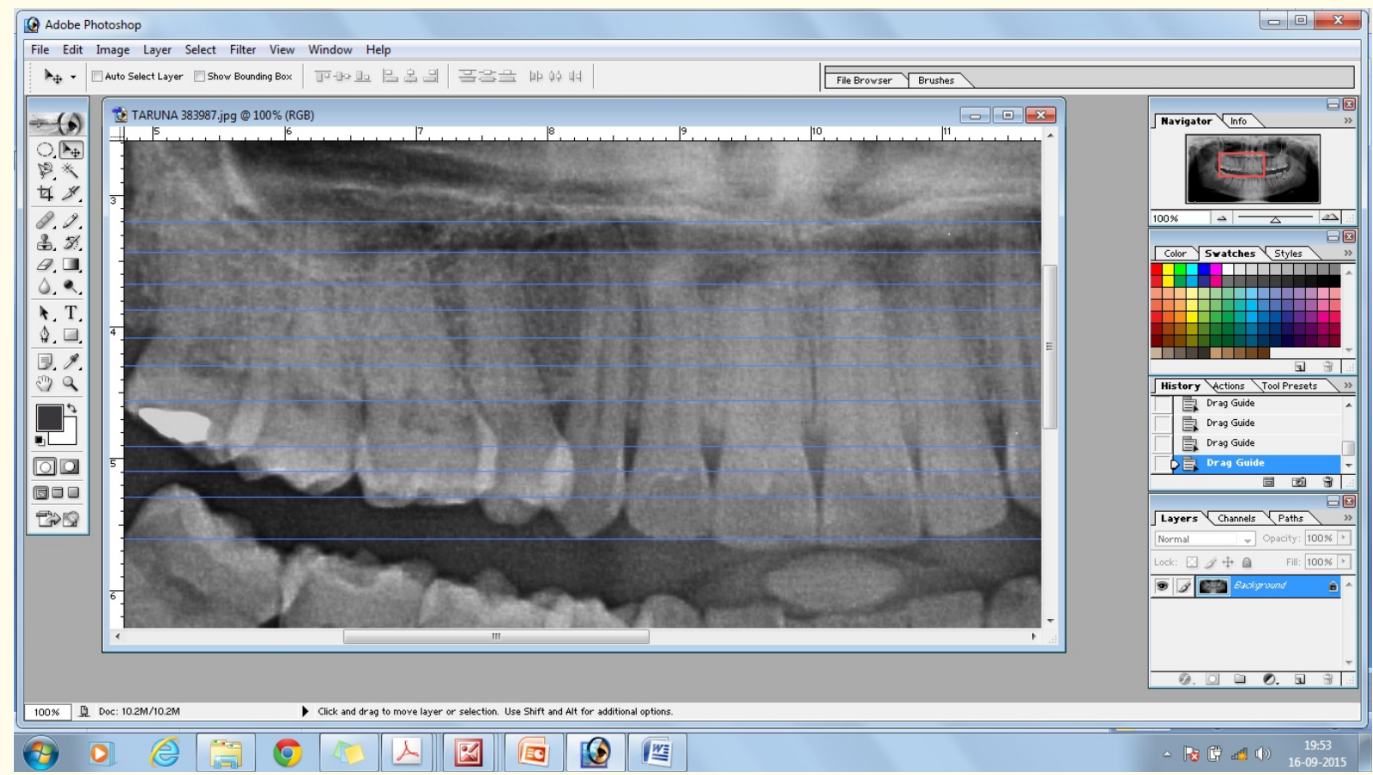

Figure 1: Horizontal lines marked on the tooth.

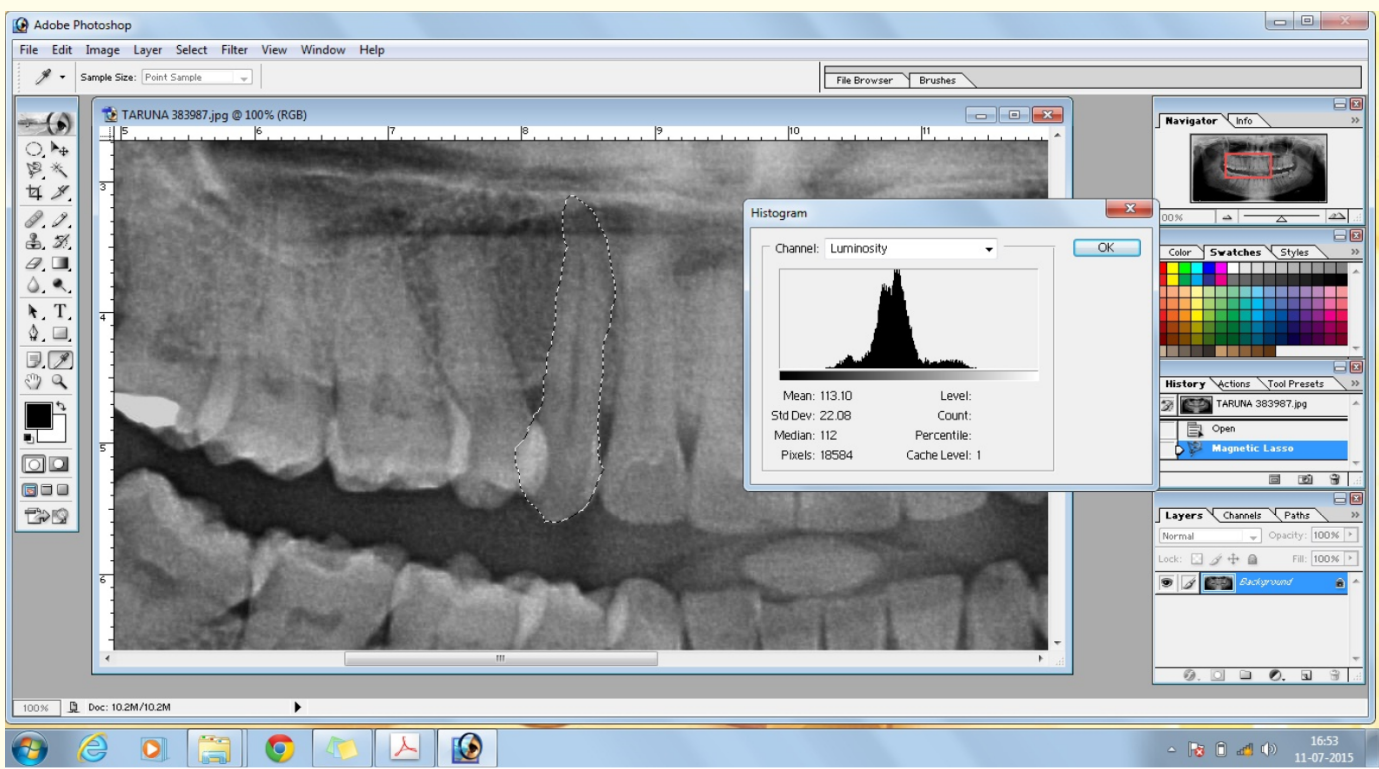

Figure 2: Tooth area obtained by connecting points. 


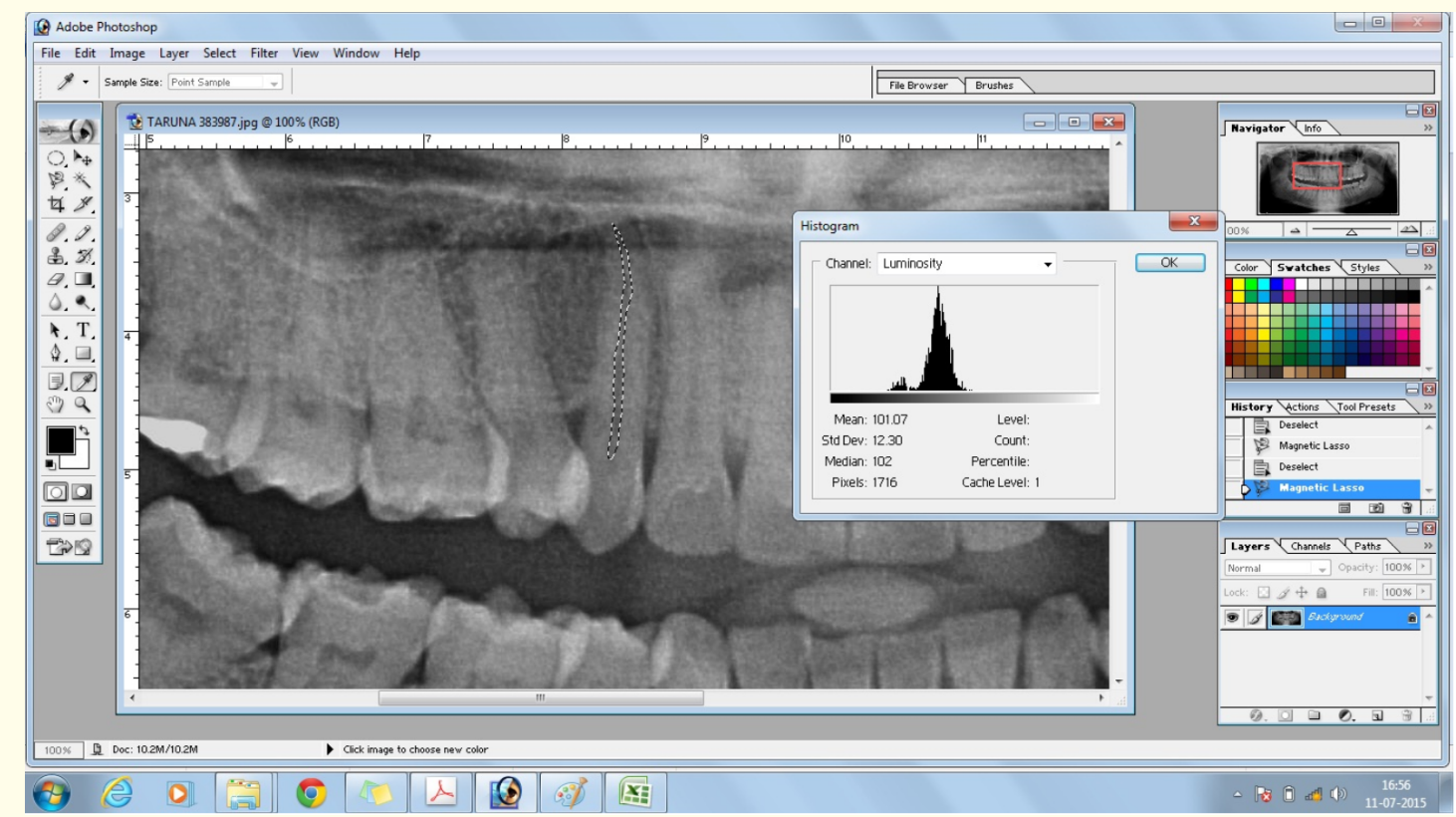

Figure 3: Pulp area obtained by connecting points

All measurements were made without prior information about personal data of the subjects. Age was calculated using the linear regression equations developed by Cameriere.,et al.[11] and Jeevan.,et al.[13] for maxillary and mandibular canines, Bebshet.,et al.[15] for mandibular canines and using Sexena's[16] and Juneja's[17] equations for maxillary canines.

The actual chronological age predicted dental age by the different methods and the difference between actual and predicted age (the 'error') was tabulated using Microsoft Office 2007 Excel spreadsheet (Microsoft Corp., Redmond, WA, USA). The absolute values of the errors for each method were tabulated and its average calculated. This is the 'mean absolute error' or MAE. The MAE depicts the average magnitude of error in the age predictions and has been used as a measure of accuracy of age estimation methods $[18,19]$. In addition to the MAE, the number/percentage of estimates with errors $< \pm 10$ years for each method were also tabulated. Errors $< \pm 10$ years is considered by some as "acceptable" in forensic age prediction [20]; it is also the interval given most often in forensic age estimation and the error usually associated with post-mortem age assessments [21]. This approach of quantifying error was used in a recent age estimation study on Indians [22] and using a similar approach here possibly allows some form of comparison of the usefulness of various age estimation methods in Indians.

\section{Results}

The paired t-test to evaluate intra- and inter-observer error revealed no significant differences ( $p>0.05$ ) (Table 2).

\begin{tabular}{|c|c|c|c|}
\hline Examination & $\mathbf{N}$ & t-Value & p value \\
\hline Intra-observer & 25 & -0.787 & 0.44 \\
\hline Inter-observer & 25 & -1.849 & 0.15 \\
\hline
\end{tabular}

Table 2: Paired t-test evaluating intra- and inter-observer variation in measuring pulp/tooth area ratio.

Age estimation using maxillary canine by Cameriere's formula produced an MAE of 11.67 years with 58/100 cases (58\%) age estimation was within \pm 10 years of actual age; Jeevan's formula produced an MAE of 10.57 years yielding "acceptable" age estimates (i.e. error $< \pm 10$ years of actual age) in 57/100cases (57\%); Sexena's formula produced an MAE of 10.68 years yielding "acceptable" age estimates in 56/100cases (56\%); Juneja's formula produced an MAE of 10.59 years revealing "acceptable" age estimates in 55/100cases (55\%) (Table 3).

Age estimation using Mandibular canine by Camerie's formula produced an MAE of 11.65 years revealing "acceptable" age esti- 


\begin{tabular}{|l|c|c|}
\hline $\begin{array}{c}\text { Name of formula } \\
\text { used }\end{array}$ & $\begin{array}{c}\text { Mean } \\
\text { absolute } \\
\text { error or MAE }\end{array}$ & $\begin{array}{c}\text { Number and percentage } \\
\text { of cases with errors < 10 } \\
\text { years i.e. acceptable" age } \\
\text { estimates }\end{array}$ \\
\hline Cameriere's formula & 11.67 years & $58 / 100$ cases $(58 \%)$ \\
\hline Jeevan's formula & 10.57 years & $57 / 100$ cases (57\%) \\
\hline Sexena's formula & 10.68 years & $56 / 100$ cases (56\%) \\
\hline Juneja's formula & 10.59 years & $55 / 100$ cases $(55 \%)$ \\
\hline
\end{tabular}

Table 3: Comparison of age estimation outcomes using maxillary canine.

mates in 58/100cases (58\%); Jeevan's formula produced an MAE of 10.12 years showing "acceptable" age estimates in $57 / 100$ cases (57\%); Bebshet's formula produced an MAE of 10.75 years yielding "acceptable" age estimates in 56/100cases (56\%) (Table 4).

\begin{tabular}{|l|c|c|}
\hline $\begin{array}{c}\text { Name of formula } \\
\text { used }\end{array}$ & $\begin{array}{c}\text { Mean } \\
\text { absolute } \\
\text { error or } \\
\text { MAE }\end{array}$ & $\begin{array}{c}\text { Number and percentage } \\
\text { of cases with errors < +10 } \\
\text { years i.e. acceptable" age } \\
\text { estimates }\end{array}$ \\
\hline Cameriere's formula & 11.65 years & $58 / 100$ cases $(58 \%)$ \\
\hline Jeevan's formula & 10.12 years & $57 / 100$ cases $(57 \%)$ \\
\hline Bebshet's formula & 10.75 years & $56 / 100$ cases $(56 \%)$ \\
\hline
\end{tabular}

Table 4: Comparison of age estimation outcomes using mandibular canine.

\section{Discussion}

Owing to the great impetus towards dental age estimation since last decade; a number of new methods for dental age assessment using different techniques viz. Polarized microscopy [23,24]/confocal microscopy [25] have been developed. Also, existing methods have been refined [1-8]. Although, most of these claim to provide relatively precise age estimates; however, major drawback of these methods is the need to extract and process teeth [23-25]. Thus, this precludes their use in livings, in forensic cases where post-mortem tooth extraction is not permitted due to legal and cultural reasons, or situations where skeletal remains are scarce and need to be preserved [1-8]. Dental radiographs play a vital role in such situations, circumventing the mentioned limitations [9-16].

The study of morphological parameters of the teeth i.e. pulp/ tooth area ratio on radiographs is considered to be more reliable method of age estimation [9-16]. It is an indirect quantification of secondary dentine deposition. Secondary dentine has been preferred since it is encased not only by harder tissue such as enamel and cementum, but also by primary dentine [9-16]. Consequently, its evaluation is considered to be an "internal examination", with the potential to eliminate the effect of environmental factors on human remains [9-16].

It was established in 1925 by Bodecker[26] that the apposition of secondary dentin correlated with age. In 1995, Kvaal.,et al. [10], presented a method for age estimation, which was based on investigation of periapical radiographs, whereas Paewinsky.,et al. [27] verified the applicability of this method on orthopantomograms. In 2004, Cameriere.,et al. [11] for the first time conducted a preliminary study to evaluate the variations in pulp/tooth area ratio (AR) as an indicator of age and their method of age estimation seems promising.They revealed linear regression between age and the AR ratio in maxillary and mandibular canines.Since canines are single rooted teeth with largest pulp area thus provides ease of analysis and are frequently present in older age groups have less chance for wear as compared to other anterior teeth; therefore they are most favored for age estimation.Cameriere.,et al. $[9,11]$ used this method on Italians, Portuguese as well as on skeletal remains in Italy. Periapical X-rays and orthopantomographs were used to image the canines which were then digitalized and used for analysis. In the present study, we sought to estimate the AR in canines (maxillary and mandibular) in anIndian population in Faridabad district of Haryana using digital orthopantomograms. Digital orthopantomograms overcomes the need for scanning and digitalization of radiographs. They also offered various advantages over traditional ones such as minimal time, ease of repeatability, accessibility, increased precision, accuracy and reliability.

The present study demonstrated good intra-observer and interobserver agreement indicating high reproducibility of the measurements. This is in accordance with studies by Cameriere.,et al. [11], Jeevan.,et al. [13], Bebshet.,et al. [15], Sexena[16], Juneja.,et al. [17].

The dental age estimation using Cameriere's formula [9,11], present study revealed a MAE of 11.67 years and11.65 years for maxillary and mandibular canines respectively. This is in accordance with study by Bebshet M.,et al. [15] who applied Cameriere's formula on Indian population and found the MAE of 11.01 years. However, Cameriere.,et al. $[9,11]$ in their original study reported error of 2.58 - 5.4 years in Italian population.

In order to enhance the age prediction Indian population specific equations were given by Jeevan et. al. [13] for both maxillary and mandibular canines, Bebshet.,et al. [15] modified the Cameriere's equation for mandibular canines and Sexena[16], Juneja.,et al. [17] modified the Cameriere's equation for maxillary canines. To 
our best knowledge, no study till date has been done to assess their applicability and compare these.

The dental age estimation using Jeevan's formula [13], present study revealed a MAE of 10.57 years and10.12 years for maxillary and mandibular canines respectively. However in their original study, Jeevan.,et al. [13] reported the error of 4.28 years for maxillary and 6.39 years for mandibular canines.

The dental age estimation using Bebshet's formula [15] for mandibular canines, present study showed 10.75 years of MAE. This is in accordance to Bebshet M.,et al. [15], who in their original study reported the MAE of 10.76 years. The dental age estimation using Sexena's formula [16] for maxillary canines, present study showed 10.68 years of MAE; whereas the authors in their original study have reported the error of 11.56 years. The dental age estimation using Juneja's formula [17] for maxillary canines, present study showed 10.59 years of MAE; whereas the authors in their original study have reported the error of 3.02 years.

The plausible cause of different error values obtained in present study from those reported in original ones may be due to a combination of factors including ethnic racial, cultural, dietary differences and variation in the pattern of secondary dentine deposition which may be relatively slow and irregularly paced in this population $[16,17]$.

Although, the study showed no recognisable difference in dental age assessed by all the methods, but Jeevan's formula revealed least MAE and slightly more percentage of cases with errors $< \pm 10$ years i.e. acceptable" age estimates than others, Therefore,Jeevan's.,et al. method is considered over other methods for the estimation of age in Indian population.

\section{Conclusion}

Within the limitations of the study, it can be inferred that Jeevan.,et al.method is more applicable for Indian population. However, further research should aim at involving larger samples which include varying geographic regions and races in order to make inferences on the method's appropriateness.

\section{Acknowledgements}

The authors acknowledge the divine presence and blessings of Late Dr. Jatinder Kumar Gupta and dedicate the whole work to him. The authors also acknowledge the writing assistance provided by Angel Medical Solutions. Authors declare that they did not receive any sponsorship and financial support for the paper.

\section{Conflict of Interest}

Authors declare that there are no financial interests or any conflict of interest.

\section{Bibliography}

1. Gupta S., et al. "Age estimation in Indian children and adolescents in the NCR region of Haryana: A comparative study". Journal of Forensic Dental Sciences 7.3 (2015): 253.

2. Limdiwala P., et al. "Age estimation by using dental radiographs". Journal of Forensic Dental Sciences 5.2 (2013): 118.

3. Chandramala R. "Application of Kvaal's Technique of Age Estimation on Digital Panoramic Radiographs". Dentistry 02.06 (2012).

4. Zaher J., et al. "Age estimation from pulp/tooth area ratio in maxillary incisors among Egyptians using dental radiographic images". Journal of Forensic and Legal Medicine 18.2 (2011): 62-65.

5. Panchbhai A. "Dental radiographic indicators, a key to age estimation”. Dentomaxillofacial Radiology 40.4 (2011): 199-212.

6. Singaraju S., et al. "Age estimation using pulp/tooth area ratio: A digital image analysis". Journal of Forensic Dental Sciences 1.1 (2009): 37.

7. Veera S., et al. "Coronal pulp biomarker: A lesser known age estimation modality". Journal of Indian Academy of Oral Medicine and Radiology 26.4 (2014): 398.

8. Ayad CE., et al. "Estimation of Age for Sudanese Adults using Orthopantomographs". Global Journal of Medical Research: Journal Dentistry and Otolaryngology 14.1 (2014).

9. Cameriere R., et al. "Age Estimation by Pulp/Tooth Ratio in Canines by Peri-Apical X-Rays". Journal of Forensic Sciences 52.1(2007): 166-170.

10. Kvaal SI., et al. "Age estimation of adults from dental radiographs". Forensic Science International 74 (1995): 175-185.

11. Cameriere R., et al. "Variations in Pulp/Tooth Area Ratio as an Indicator of Age: a Preliminary Study". Journal of Forensic Sciences 49.2 (2004): 1-3.

12. Bagh T., et al. "Age Estimation using Cameriere's Seven Teeth Method with Indian Specific Formula in South Indian Children". International Journal of Advanced Health Sciences 1.2 (2014): 1-10. 
13. Jeevan M., et al. "Age estimation by pulp/tooth area ratio in canines: Cameriere's method assessed in an Indian sample using radiovisiography". Forensic Science International 204.1-3 (2011): 209.e1-209.e5.

14. Babshet M., et al. "Age estimation in Indians from pulp/tooth area ratio of mandibular canines". Forensic Science International 197.1-3 (2010): 125.e1-125.e4.

15. Babshet M., et al. "Age estimation from pulp/tooth area ratio (PTR) in an Indian sample: A preliminary comparison of three mandibular teeth used alone and in combination". Journal of Forensic and Legal Medicine 18.8 (2011): 350-354.

16. Saxena S. "Age estimation of indian adults from orthopantomographs". Brazilian Oral Research 25.3 (2011): 225-229.

17. Juneja M., et al. "Age estimation using pulp/tooth area ratio in maxillary canines A digital image analysis". Journal of Forensic Dental Sciences 6.3 (2014): 160-165.

18. Spalding KL., et al. "Age written in teeth by nuclear tests". Nature 437.15(2005): 333-334.

19. Cameriere R., et al. "Accuracy of age estimation in children using radiograph of developing teeth". Forensic Science International 176.2-3 (2008): 173-177.

20. Solheim T., et al. "Dental age estimation of Norwegian adults: a comparison of different methods". Forensic Science International 16.1 (1980): 7-17.

21. Reppien K., et al. "Evaluation of post-mortem estimated dental age versus real age: A retrospective 21-year survey". Forensic Science International 159 (2006): S84-S88.

22. Acharya A., et al. "Effectiveness of Bang and Ramm's formulae in age assessment of Indians from dentin translucency length". The International Journal of Legal Medicine 123.6(2009): 483488.

23. Kasetty S., et al. "Dental Cementum in Age Estimation: A Polarized Light and Stereomicroscopic Study". Journal of Forensic Sciences 55.3 (2010): 779-783.

24. Kaur P., et al. "Estimation of age based on tooth cementum annulations: A comparative study using light, polarized, and phase contrast microscopy". Journal of Forensic Dental Sciences 7 (2015): 215-221.
25. Pundir S., et al. "Estimation of age based on tooth cementum annulations using three different microscopic methods". Journal of Forensic Dental Sciences 1(2009): 82-87.

26. Bodecker CF. "A consideration of some of the changes in the teeth from young to old age". Dental Cosmos 67 (1925): 543549.

27. Paewinsky E., et al. "Quantification of secondary dentin formation from orthopantomograms. A contribution to forensic age estimation methods in adults". The International Journal of Legal Medicine 119 (2005): 27-30.

\section{Assets from publication with us}

- Prompt Acknowledgement after receiving the article

- Thorough Double blinded peer review

- Rapid Publication

- Issue of Publication Certificate

- High visibility of your Published work

Website: www.actascientific.com/

Submit Article: www.actascientific.com/submission.php Email us: editor@actascientific.com

Contact us: +919182824667 